\title{
Errores en las referencias bibliográficas de la producción académica: un estudio de caso
}

\section{Cintia Braga Ferreira}

Bibliotecária de la Facultad de Filosofía, Ciências y Letras de Ribeirao Preto/USP (Brasil)

Maria Bernadete Malerbo

Bibliotecária de la Revista Latino-americana de Enfermería-EERP/USP (Brasil)

Márcia Regina Silva

Centro Universitário Barão de Mauá (Brasil)

\subsection{Resumen}

Análisis de los errores en las citas bibliográficas de las tesis de maestría presentadas en 1999 y 2000 en el curso de postgrado interunidades de bioingeniería de la USP. Se realizó la selección aleatoria de una muestra de 280 referencias. Los datos fueron examinados en MEDLINE, PubMed, Web of Science y Literatura Latinoamericana y del Caribe en Ciencias de la Salud (LILACS), y se excluyeron de la muestra las referencias de documentos no catalogados. El 27,6\% de las referencias examinadas contenían al menos un error. De los errores que se encontraron se clasificaron como mayores aquéllos susceptibles de retrasar la localización de los documentos (70\%) y como menores el resto (30\%). El índice de referencias incorrectas fue bajo, aunque la mayor parte de los errores encontrados compromete la función del documento como fuente de información, reflejando la necesidad de que se cuide mejor el proceso de revisión de las disertaciones.

Palabras clave: Tesis doctorales. Tesis de maestría. Referencias bibliográficas. Calidad. Evaluación.

\subsection{Abstract}

Analysis of the error rate of bibliographic citations in the dissertations presented for a master degree in bioengineering at University of São Paulo from 1999 to 2000. A random sample of 280 references was verified in MEDLINE, PubMed, Web of Science and Latin American and Caribbean Health Science Literature (LILACS), excluding non-indexed materials. The accuracy of the authors names, title and source items was determined. The errors founded were classified as minor when they did not delay the retrieval of the document and

Scire. $9: 1$ (en.-jun. 2003) 133-138. 
major when they did. From the 163 references verified $27.6 \%$ had at least one citation error. 60 errors in total were found: $70 \%$ major and $30 \%$ minor. The error rate was low (27.6\%), but the majority of them can delay the document retrieval and so compromising their quality as a source of information. More attention is required from reviewers of the texts. The librarians must contribute with the authors by improving the citations accuracy through technological information sources. According to a recent paper, the length of reference list is a risk factor for citation errors. It is necessary to consider this factor in order to improve the accuracy of the references.

Keywords: Doctoral dissertations. Master dissertations. Bibliographic references. Quality evaluation.

\section{Introducción}

En el campo de la sociología de la ciencia, las referencias bibliográficas y las citaciones son importantes en la divulgación de los resultados y en la recuperación de información, pues los errores y inexactitudes comprometen los resultados.

Dentro de un contexto de cambio en el proceso de investigación científica, el conjunto de referencias bibliográficas utilizadas reflejan la contextualización teórica del trabajo, además de ser utilizadas como fuente de información. Como afirma Reyes (1998), "las referencias bibliográficas reflejan una de las características de la investigación científica: cada nuevo trabajo se apoya en el trabajo de predecesores del campo del conocimiento". De esa forma, esta sección de los documentos científicos demuestra su importancia al establecer relaciones de conceptos. La selección adecuada de la bibliografía citada es indispensable para el fortalecimiento del trabajo y credibilidad del manuscrito (Reyes, 1998). Lichter (1989) afirma que las referencias bibliográficas de un artículo son tan importantes para la determinación de la calidad del trabajo como la metodología utilizada, los resultados obtenidos y su discusión. Baron (2001), en una carta enviada a Lancet, comenta el consejo recibido al iniciar su trabajo de examinador externo de tesis y disertaciones: seleccionar aleatoriamente y verificar la exactitud de una docena de referencias bibliográficas; si se detecta un error, se verifica otra docena. Cuando se observa que un candidato tiene errores persistentes en las referencias, casi siempre surgen otros errores inaceptables en el cuerpo del trabajo.

La comunidad científica registra y divulga su conocimiento a través de diversos tipos de documentos, entre los cuales destacan disertaciones y tesis de los cursos de postgrado. La producción de disertaciones y tesis en las universidades brasileñas es obligatoria para la obtención de los títulos de postgrado, así como su defensa pública ante una comisión. Alves (1992) afirma que, tras su defensa, "el destino de la gran mayoría de las tesis y disertaciones es envejecer en los estantes

Scire. $9: 1$ (en.-jun. 2003) 133-138. 
de bibliotecas universitarias" y que los usuarios no las consultan a no ser como fuentes complementarias de información bibliográfica.

Utilizar fuentes bibliográficas de información es el primer paso para la producción del conocimiento científico. A través de las fuentes consultadas, se obtienen los datos necesarios para el desarrollo del trabajo, proporcionando la revisión bibliográfica sobre determinado tema, estableciendo el estado de la cuestión en determinada área de conocimiento. Entre los diferentes tipos de fuentes bibliográficas podemos destacar las referencias bibliográficas citadas en un documento. Las referencias bibliográficas, como se ha afirmado anteriormente, además de indicar el fundamento teórico de los trabajos, también ofrecen auxilio para la producción de nuevos textos a otros autores que consultan y evalúan el documento. Otro tipo de fuente bibliográfica es la bibliografía, que consiste en un elemento de apoyo al investigador y a otras personas interesadas en la búsqueda e identificación de libros, revistas y otros tipos de material documental útiles para su investigación. A través de la bibliografía es posible identificar el camino recorrido por el autor, localizar el material utilizado, divulgar trabajos desarrollados por otros autores e identificar los documentos e informar sobre sus contenidos.

La inexactitud de las citaciones refleja el descuido académico del autor, compromete la calidad del trabajo y puede perjudicar la recuperación de la información. Rudolph y Brackstone (apud Taylor, 1998) afirman que los académicos se muestran poco interesados en el tema hasta el momento en que dichos errores comprometen o impiden el desarrollo de sus investigaciones. Taylor (1998) trata en su artículo de los efectos prácticos de la inexactitud de las referencias bibliográficas. Además de comprometer, en algunos casos, la recuperación de documentos originales, los errores en los nombres de los autores impiden que les sea otorgado el crédito debido y que otros trabajos del mismo autor sean recuperados. Los índices de citación utilizan la lista de referencias bibliográficas como fuente primaria de información, aunque las listas con inexactitudes generan información inexacta. Taylor (1998) resalta que algunas instituciones basan sus criterios de cancelación o subscripción de revistas en el número de citaciones que determinado título recibe en los trabajos académicos producidos por la institución. Las incorrecciones bibliográficas comprometen tanto la dinámica del conocimiento científico como sus servicios de apoyo.

\section{Objetivo}

El objetivo de este trabajo es verificar los errores de las citaciones en las disertaciones presentadas en 1999 y 2000 para la obtención del grado de maestría en el Curso de postgraduación interunidades Bioingeniería de la Universidad de San Paulo, Brasil. 


\section{Metodología}

Para el estudio, se seleccionó el Programa de Postgraduación Interunidades en Bioingeniería (PPIB) del Departamento de Ingeniería de Materiales de la Escuela de Ingeniería de San Carlos y el Departamento de Ortopedia de la Facultad de Medicina de Ribeirão Preto de la Universidad de San Paulo (USP). En el PPIB fueron defendidas hasta el mes de agosto de 2002 noventa disertaciones de maestría, de las cuales cuarenta y seis fueron defendidas en el periodo 1998-2002. Para el estudio se optó por trabajar con las tesis producidas en los años 1999 y 2000.

Seguidamente se seleccionó aleatoriamente una muestra de 280 referencias. El tamaño de la muestra fue determinado a través del método de Tamaño de Célula de Galtung (Losee Jr. y Worlev, 1993). Los datos fueron verificados en las bases de datos automatizadas Web of Science, PubMed, MEDLINE, LILACS, etc. De la muestra se excluyeron 117 referencias de documentos no indizados, el $41,78 \%$, consistentes en libros, capítulos de libro, comunicaciones en congresos y tesis. Los datos investigados fueron el nombre de los autores, el título del artículo y la fuente de referencia - título de la revista, año de publicación, número de volumen y paginación - . Se consideraron ítems esenciales para localización de los documentos el nombre del autor, el título de la revista, el volumen, el número y el año de publicación. El título del artículo y paginación no fueron considerados esenciales para la recuperación del documento.

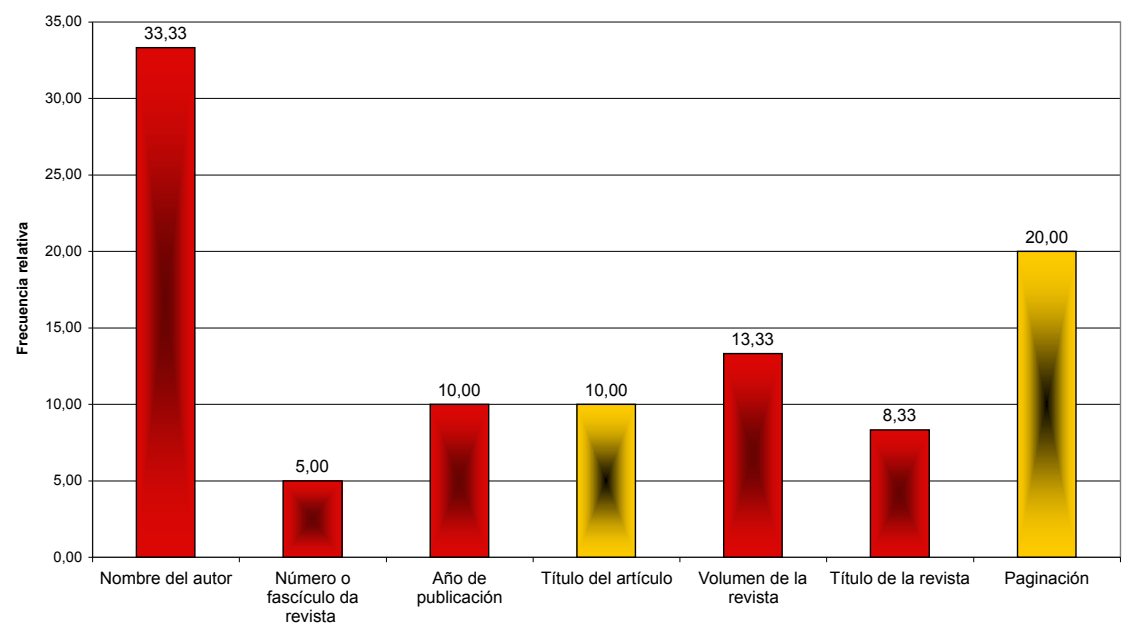

Fig. 1. Gráfico de la distribución de los errores detectados en las referencias bibliográficas analizadas.

Scire. $9: 1$ (en.-jun. 2003) 133-138. 


\section{Resultados}

Se verificaron 163 referencias bibliográficas -el 58,22\% de la muestra seleccionada - . De acuerdo con Noronha (1998), en las tesis y disertaciones predominan los artículos de revistas, "pero en menor escala comparados con la proporción citada en los propios artículos". De las 163 referencias, 45 (27,6\%) presentaron por lo menos un error; de ese total $29(64,45 \%)$ tenían una única incorrección y $16(35,55 \%)$ dos o más datos bibliográficos incorrectos. Los errores encontrados fueron clasificados en dos grupos: graves, aquéllos susceptibles de retrasar la localización del documento y que resultaron ser el 70\%; y leves, aquéllos que no, y que constituyeron el $30 \%$ (fig. 1). Los errores encontrados comprometen seriamente la función del documento como fuente de información, revelando la necesidad de un mayor cuidado en el proceso de revisión de las tesis.

\section{Consideraciones finales}

De acuerdo con Noronha (1998, p. 73) "las disertaciones y tesis representan el inicio de actividades académicas y científicas de sus autores, por lo tanto, esos trabajos deben ser realizados con el rigor científico exigible a un trabajo de investigación". Los programas de postgrado deben ser exigentes en la evaluación de los trabajos presentados, determinando las normas y reglas que deben seguirse durante la fase de organización del trabajo científico.

Entre las diversas hipótesis barajadas para explicar la alta tasa de errores destacan la escasa formación y entrenamiento de los autores en la normalización y presentación del trabajo científico y en la conversión de las referencias de una norma a otra; y la poca importancia dada las referencias bibliográficas y a la citación de trabajos no leídos. El bibliotecario de referencia puede desempeñar un importante papel, orientando y revisando los datos presentados, y minimizando, de esa forma, el número de errores en las referencias bibliográficas. Algunas revistas internacionales proceden a la evaluación de la bibliografía citada en los manuscritos presentados para su revisión previa. Los errores se presentan a los autores para su corrección. Desgraciadamente, no ocurre lo mismo con las disertaciones y tesis, pues los autores no se preocupan de dicha verificación. Sekerak et al. (1995) concluyeron que los bibliotecarios podrían contribuir significativamente a mejorar la exactitud de las citas con el uso de las tecnologías de información.

La divulgación de esta investigación y de otras que prueban la importancia de la normalización de los trabajos científicos es de gran valor para una comunidad científica que busca credibilidad y reconocimiento para su producción intelectual. En el futuro, se prevé continuar las investigaciones, ampliando el muestreo y abordando también otros campos del saber. 


\section{Referencias}

Alves, A. J. A. (1992). "Revisão da bibliografía” em teses e dissertações: meus tipos inesquecíveis. // Cadernos de Pesquisa. 81 (Maio 1992) 53-60.

Baron D. N. (2001). Accuracy of references in theses. // Lancet. 357:9250 (Jan. 2001).

URL: http://www.thelancet.com/journal.isa . Consultado: 2002-06-04.

Lichter, P. R. (1989). Checking citations. // Ophthalmology. 96: 8 (Aug 1989) 1131-2.

Losee Jr., R. M.; Worley, K. A. (1993). Research and evaluation for information professionals. San Diego: Academic Press, 1993.

Noronha, Dayse Pires (1998). Análise das citações das dissertações de mestrado e teses de doutorado em saúde pública (1990-1994): estudo exploratório. // Ciencia da Informação. 27:1 (1998) 66-75.

Reyes, H. (1998). Las referencias en artículos publicados en revistas biomédicas. // Revista Médica de Chile. 129:4 (abr 1998). URL: htttp://www.scielo.cl/scielo. php?script=sci_serial\&pid=0034-9887\&lng=es\&nrm=isop. Consultado: $2002-05$ 04.

Sekerak, R. J. (1995). Librarians contract to provide bibliographic support for a new medical text. // Bulletin of Medical Library Association. 83:1 (Jan. 1995) 93-95.

Taylor, M. K. (1998). The practical effects of errors in reference lists in nursing research journals. Nursing Research. 47:5 (Sep.-Oct. 1998) 300-303. 